\section{A REMARKABLE CASE OF MYCETOMA.}

BY SURgeon-MaJoR WM. KEITH HATCH, F.R.C.S. ENG., M.B., C.M. ABERD. ;

AND

Surgeon-Captain F. L. ChILdE, M.B. Lond., M.R.C.S. KNG.

PHYSICIAN TO THE SIR JAMSETJEE JEEJHEEBHOY HOSPITAL, BOMBAY.

THE patient was a pure African about thirty-five years of age, and was admitted into the Sir Jamsetjee Jeejheebhoy Hospital, Bombay, on Jan. 21st, 1894, in a weak and emaciated condition. He was then under the care of SurgeonMajor Hatch. He had spent the early part of his life in England at a home for Asiatics, and went to sea at first as a cabin boy and later served as fireman on several ships; he lived on shore at intervals in England and at foreign portspiz., America, China, France, India, and Africa. He stated

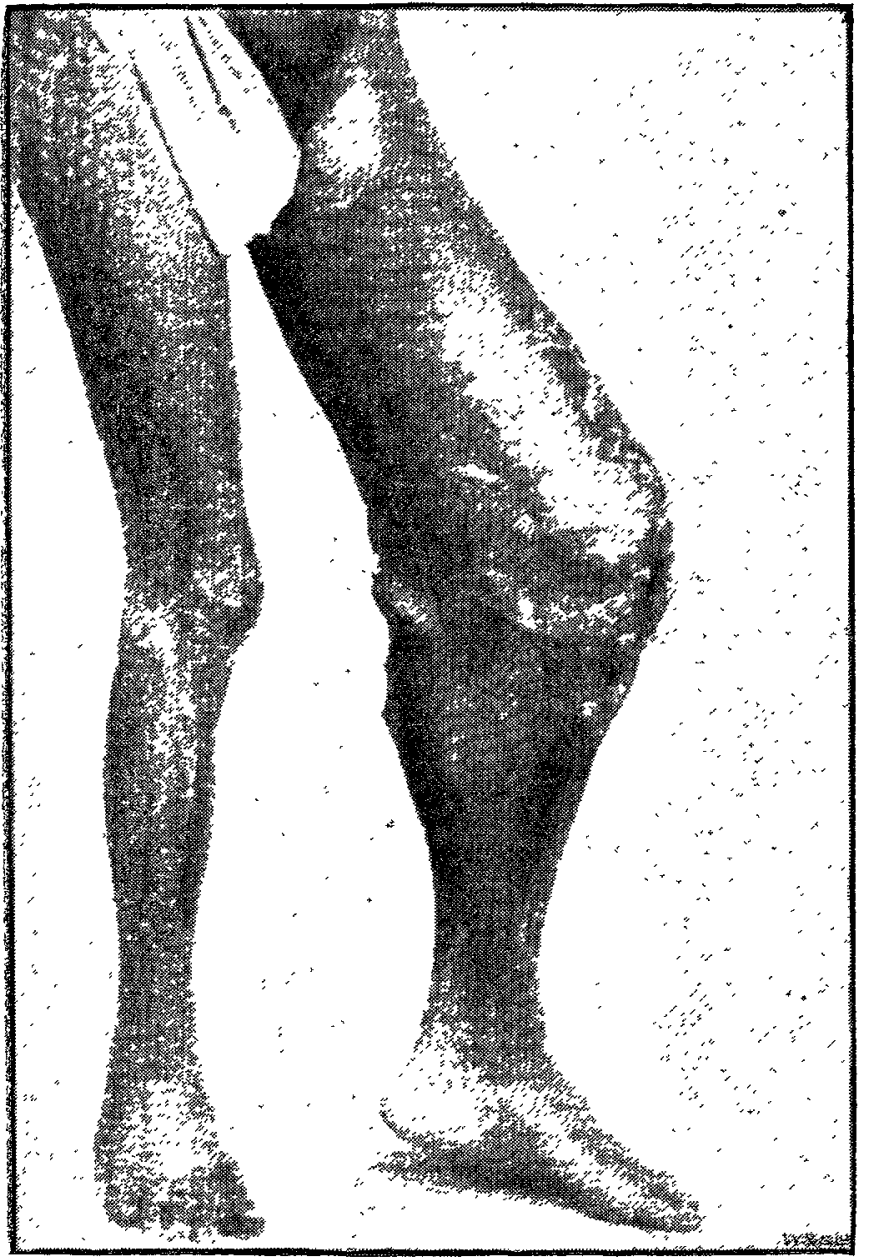

that he had never gone inland, and rarely stayed more than a week at any port. He had never suffered from any illness before, had no family, and believed that he came originally from Zanzibar. His illness commenced a y ear previously after a fall. He was working on deck and fell down a companion ladder into the hold about $10 \mathrm{ft}$. or $12 \mathrm{ft}$. According to the patient's statement his left patella was displaced to the inner side, and next morning the left knee became much swollen. After a few days he returned to work, although there was considerable pain on the outer side of the knee; the knee and thigh steadily increased in size during the next six months; at length, while on a voyage from China to Ifngland, he could work no longer and asked the captain to leave him at Aden, where he was admitted into hospital. He then noticed for the first time the appearance of small swellings like blisters over the left thigh and knee; these burst and discharged pus; small seed-like bodies were also noticed in the discharge. After remaining at Aden four months he left for Bombay. During this period the swelling of the limb increased and he became unable to walk. He was admitted into the Sir Jamsetjee Jeejheebhoy Hospital,
Bombay, under Surgeon-Major Hatch's charge on Jan. 21st, 1894. The patient was a strongly built man in poor condition: his skin was of an ashy colour, as seen in sick negroes. He could speak broken English, but was extremely ignorant; the mucons membrane was very anæmic; the muscles were also much atrophled. He was quite unable to walk owing to pain and the unwieldy size of the left lower extremity; this from the junction of the upper and middle third of the thigh to the middle third of the leg was enormously enlarged, having ronghly the appearance of elephantiasis. The skin over this part was darker than that of the body generally, and the limb felt hot; the enlargement appeared almost uniform, but was most marked at the knee. The surface, more especially in front and on the outer side, was covered with soft nummular swellings, rather larger than a shilling and slightly convex. On the summit of some were dried scabs; others had a cicatricial appearance; while otbers, again, discharged blood and pus from one or more sinuses. These swellings could be easily ruptured with a probe, when a small quantity of pus, with seed-like bodies and balls, varying in size from a millet-seed to a pea, conld be removed.

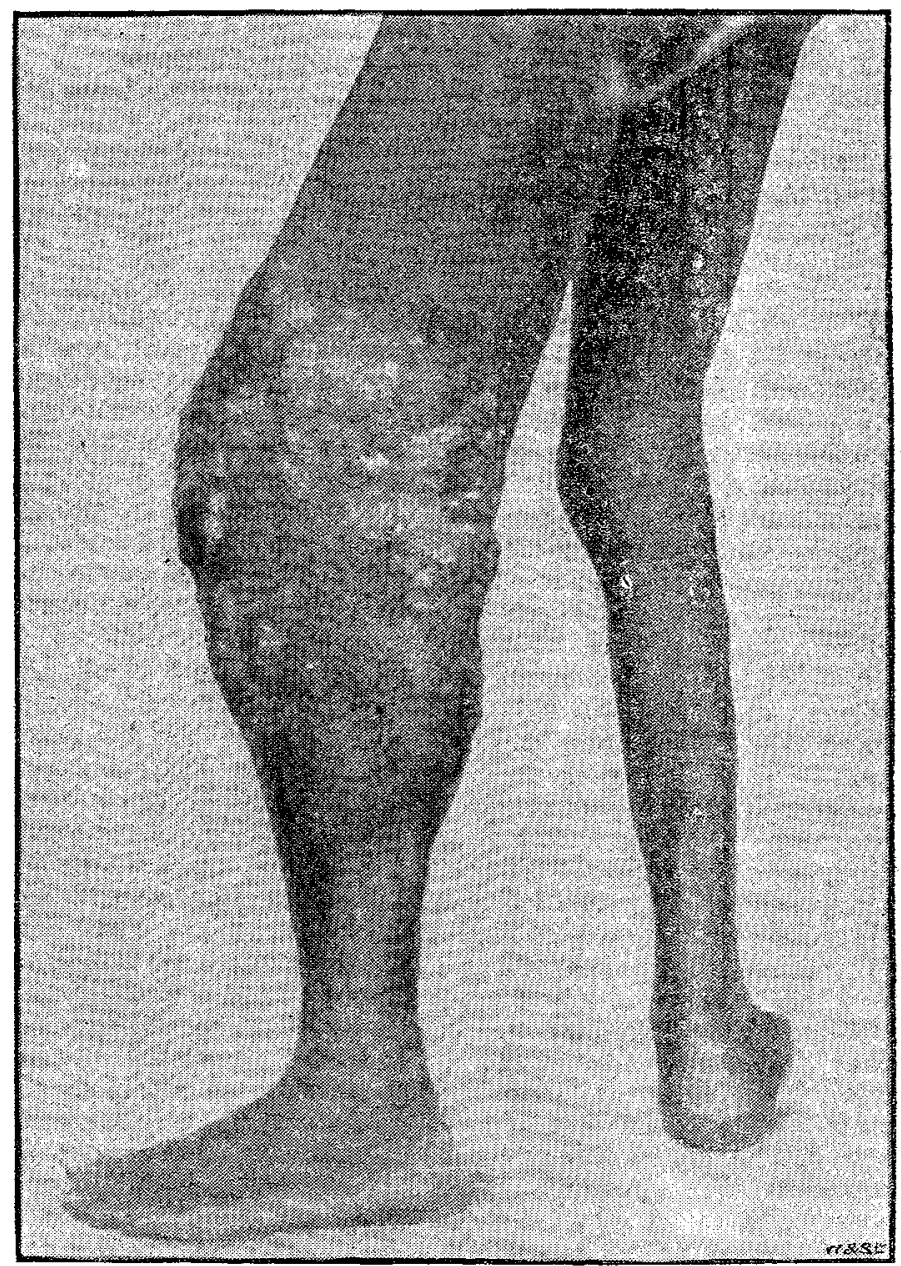

The cavities containing these bodies appeared to be quite superficial, the probe only passing for a quarter of an inch ; subsequently a sero-mucous discharge contınued to drain from the openings and rapidly decomposed. The diseased part was fairly firm, but softest over the muscles of the calf and the inner side of the knee, firm and brawny over the joint and immediately above it. The patella and tuberosities of the tibia could be felt. The whole discased area was tender and especially painful on the outer side, and pitted on firm pressure; the lower part of the limb was not odematoug. In the left groin there was a soft, slijhtly movable, and nodular swelling, over which the kkin was studded with small bluish-black nodules; there were no openirgs in these. Similar swellings could be felt in the lower part of the abdomen above Poupart's ligament and aling the course of the left external iliac vessels ; the largest was abont the size of a hen's egg. The supra-trochlear glands and those of the neck were somewhat enlarged. Digestion was derarged, but the organs generally appeared healthy. The patient complained chiefly of severe abdominal pain in the epigastric region, but although the abdominal muscles were extremely lax nothing 
could be felt to account for the pain, which was chiefly nocturnal and prevented sleep; on this account be begged earnestly for ampatation of the limb, which he supposed to be the cause of the pain, but Surgeon-Major Hatch could not promise any relief by operation. The limb was wrapped in lint soaked in carbolic acid, and opinm was given internally, which relieved him a good deal, especially if the bowels were kept open. The patient began to lose flesh rapidly after admission and aged very much; his hair began to turn grey, while the limb diminished in size. He became extremely disappointed that no operative treatment was carried out, and begged for something to be done. SurgeonMajor Hatch determined to ascertain the condition of the abdominal glands, and with the assistance of SurgeonCaptain Quicke and Mr. Masina he opened the abdomen on Feb. 13th by a median incision from an inch below the umbilicus to the pubes. About a pint of serum escaped from the peritoneal cavity, and the diseased glands were then carefully removed from the neighbourhood of the iliac vessels. They were dark in colour, resembling kidneys, and easily separated. The largest was two inches long. Examination of the first specimen showed no fungus, but a quantity of black pigment. After closing the abdomen the patient was removed to the ward, when he became extremely weak. He rallied from this condition, bat died on the night of the 15th, the temperature falling to $96^{\circ} \mathrm{F}$.

Report of necrnpsy niade by Surgeon-Captain CHILDE on Feb. 16th, 1894 (twenty-two hours after death).-Rigor mortis had passed off. The body was that of an African, well built but thin, and the muscles wasted; the skin was dark and the hair woolly. The left foot and ankle were normal; at halfway up the leg the swelling began, increased, was greatest at the knee, and extended to the middle of the thigh, where it stopped; it was a general enlargement, having generally the shape of the limb, present both on the extensor and flexor surfaces, though greatest about the knee and popliteal space; thus it had a slightly fusiform appearance. The swollen part had rather a firm feel, but was softer in parts and pitted somewhat on pressure. It measured $23 \frac{1}{2}$ in. round the knee, 22 in. just above the knee, and 18 in. just below it. Numerous nodules were present on the swollen part, chiefly on the extensor surface of the knee, but also on the front of the swollen leg and thigh, and at the inner and back part of the knee. They were as large as a shilling, though sometimes less, and scabbed over, some being flat, others of a raised tubercular shape. On removing the scabs sinuses were seen, from which sanio-purnlent matter escaped, containing small flat and round yellow bodies-the yellow variety of mycetoma. There were also some sinuses open without scabs, but none of them admitted a probe more than an inch or so. There was a similar swollen part, also pitting on pressure, in the left inguinal region, with similar sinuses and dried scabs and nodules, and the glands, both inguinal and femoral, were enlarged. The leg was bisected vertically through the femur and tibia. The bones were normal except that there were some osteophytes on the inner condyle of the femur, and the knee-joint, with this exception, was quite healtby; the large arteries, veins, and nerves were traced and fourd to be normal. The muscles within the limits of the swollen part were completely disorganised and for the most part indistinguishable, so that they could not be traced; they had broken down into a large anfractuous cavity, of which the walls were most irregular and stained quite black, being composed of the remains of broken-down muscular tissue. The cavity contained thin watery fluid of a dark colour, having an oily feel; and floating in it were many dark-black shreds of tissue, of irregular shape, oilglobules and a large amount of the yellow fungus. The fungus had a slightly reddish tinge on the surface, some of it being in small particles, some collected into masses as large as peas and small marbles. The skin and subcutanemus tissues were thickened by one-third owing to chronic ir flammation, and the sinuses were found to lead down to the cavity described above ; there was no evidence of inflammation or suppuration abont the disorganised muscles, and in fact the only signs of inflammation were found in the thickened cutaneous structures and the sinuses with their sanio-purulent contents. The inguinal ard femoral lymphatic glands of the left side were enlarged but not markedly altered in consistency; they varied from the size of a walnut to that of a hazel-nut, and on the surface they were of a dark, reddish-brown tint. On section the same colouring was seen scattered about, so that the section had a mottled appearance, but markedly collected in the cortex and periphery of the gland, thus giving the brown surface-colour mentioned above; in some of the glands the fungus was found in small, rounded, yellowish masses of the size of a millet seed. each mass lying loose in a cavity slightly larger than itself. The glands along the left iliac arteries, as well as some of the mesenteric glands, were enlarged, and presented the same changes as the inguinal, except that the fungus itself was not found in any of them. The operation wound was examined, and found to be quite healthy, and there were no signs of peritonitis | either local or general. Of the other organs only the liver and spleen were abnormal. The liver weighed 1305 grammes; it was rather large, and of a light yellow colour; its surface was studded with small nodules. On section it showed a general growth. of soft, young, connective tissue-in fact, it was in the condition of early cirrhosis. The bile apparatus was normal, and dark bile was found in the gall-bladder. The spleen weighed 320 grammes; it was also large, the capsule was thick and white, looking like cartridge-paper; on section the trabeculæ were seen to be enlarged and the spleen pulp was firmer and darker than normal, the organ being in the condition of the rather chronic "malarial spleen." The right kidney weighed 205 grammes, the left 190 grammes, both being rather pale and large, but otherwise normal. The right lung weighed 550 grammes, the left 498 grammes; both were normal. The heart weighed $3 \varkappa 5$ grammes; it was rather large, but normal. The pancreas, adrenals, testicles, peritoneum, and brain were normal. All these organs were carefully examined for the mycetoma fungus, but it was not found. The blood was thin, watery, and deficient in quantity, and from this cause all the organs were paler than usual.

Mioroscopical examination. - The fluid from the cavity formed by the broken-down muscles showed many oil globules, much amorphous granular matter, and many small shreds of tissue studded with coal-black particles. There were also very many small yellow particles of the colour of blood pigment, some round, others of irregular shape; and other, but fewer, particles of a shining and almost translucent, reddish-pink appearance. These latter were more uniformly rounded than those described above and varied greatly in size. The fluid also showed much of the fangus with its characteristic stellate rays; no pus corpuscles were seen. The black shreds of tissue became colour. less on being soaked in alcohol and also after treatment with sodium bypobromite, but the yellow and pink particles were unaltered by these reagents. The inguinal glands showed $a$ large deposit of small yellow particles in all respects similar to those found in the fluid described above. These pigment particles were chiefly situated in the cortical portion of the gland so as to form a coloured band at its periphery, but they were also found freely distributed through the medullary portion. In addition many of the glands contained the fungus in small particles both in the cortex and medulla, and the gland tissue around the fungus was much disorganised, broken up, and granular, so that each fungus particle was lying in a small cavity; but no relationship was made out between the fungus and the pigment particles. The glands along the left iliac arteries and some of the mesenteric glands also showed the pigment deposit, but no sections of these glands were found to contain the fungus. When the sections of gland were treated with ferro-cyaride of potassinm and acetic acid the coloured particles in them at once gave the Prussian blue reaction ; whereas after treatment with ferri-cyanide of potassium and acetic acid the particles only showed a greenish tint after about half an hour. The fungus itself was also examined. In the fresh state each fungus particle was seen to be surrounded by a ring of closely set, fine acicular rays ; but after treating the fungus with alcohol or ether many of these rays disappeared, and then some delicate filaments could be seen, some of which were linear and others of a club shape. Similarly in sections of lymphatic gland containing the fangus-all of which were prepared by the paraffin method-many of the fine acicular rays had disappeared, having probably been dissolved by the alcobol used in the process of $\mathrm{q}$ reparation. (These fine rays were taken to be the fat crystals described by Vandyke Carter and Cunningham.)

Bacteriological examination.-Inoculations of the fungus particles were made in broth on Jan. 26th, and again on glycerin-agar on Feb. 15th, but up to March 9th no growth had occurred in either case. A mouse was inoculated with a particle beneath the skin of one flank. The animal was quite healthy and active up to March 9th. The liver, in sections, showed the 
ordinary characters of early atrophic cirrhosis ; there was no fungus.

Two other specimens of mycetoma were also examined. One, an amputated foot, was kindly sent by Surgeon-Major Parakh. Sections of a muscle were cut from this specimen, and they showed the muscle to be excavated so that only a shell remained, whilst within the cavity the fungus was found. Many round cells of inflammation were found around the fungus, and the muscle was greatly degenerated. The other specimen, also a foot, was sent by Dr. Nanavati of Ahmedabad. Sections of a subcutaneous fungus nodule were cut from this, and showed characters similar to those described above.

I have to acknowledge the assistance of Dr. N. F. Surveyor of Bombay. The sections were prepared by the parafin method, fixed with Mayer's albumen, and stained with various reagents.

Remarks by Surgeon-Major HATCH.-I have seen abont fifty cases of mycetoma in all stages in the foot, lower part of the leg, and hand. Progress has always been extremely slow, painless, and the bones invariably affected and reduced to a papery condition. Diagnosis has never presented much difficulty, except quite in the early stage when commencing in the big toe or hand. The present case is anique in my experience-the great size of the $\operatorname{limb}$, the numerous projections on the surface, the infiltrated condition of the soft parts (giving a resemblance to elephantiasis), the very rapid progress, and the absence of bone affection being especially remarkable. Secondary deposit in the glands of the groin is not uncommon, but I have never before met with enlargement of the pelvic glands. Unfortunately, the patient being so ignorant, it was impossible to get any clue as to the origin of the disease. The post-mortem notes were made by Surgeon-Captain Childe and Dr. Surveyor, who has made some valuable researches on mycetoma.

\section{THYMOL AS AN ANTHELMINTIC.} BY PROSPERO SONSINO, M.D., OF PISA.

THE history of a large number of the substances from time to time proposed as remedies is generally the following. A certain drug is suggested as a remedy for a particular disease; after some hesitancy its virtues are extolled by uumerous observers from all parts of the medical world, but as yet only as against the original disease. Then its reputed efficacy is little by little extended to many other ailments, and in a short time it is recommended in a large number of cases and diseases. Afterwards a reaction against its use arises, and its drawbacks are pointed out; and, lastly, after a long time has elapsed, its true therapeutical place is settled on sure ground, provided it does not eventually fall into complete disuse and oblivion. It seems that thymol is now jassing through this common ordeal. Thymol was used first by Bozzolo in 1880 for ankylostomiasis. After some wavering its efficacy was confirmed by many practitioners, and it is now regarded as the best remedy against ankylostomiasis, so that it has generally superseded liquid extract of male fern, the only other remedy which for some time could compete with it. But the efficacy of thymol in the treatment of ankylostomiasis was not yet well confirmed when it was already being tried in other ailments, and especially as a general anthelmintic; and now, if the assertions of some hasty eulogisers are to be trusted, it is the best remedy against all intestinal worms, tænia included, and also against the hæmatozoon filaria sanguinis hominis. I think it reflects great credit on Surgeon-Lieutenant-Colonel Crombie ${ }^{1}$ of Calcutta to have settled that about ten grammes per diem of thymol, given for three successive days, have not availed to cause the disappearance of the embryo filarix from the blood, contrary to what has been asserted by others-viz., that daily doses of some fraction of a gramme of this same drag, taken for a certain number of days, were sufficient to cure radically filarial disease. But it seems to me that Surgeon-Lieutenant-Colonel Crombie has been too hasty in asserting that thymol, while inefficacious as against filaria sanguinis, is effective against the intestinal worms, tænia

1 The Layces, Oct. 13th, 1894. included. I hope to obtain permission to record in THF LANCET the result of my experience on this subject.

Whilst I have had numerous brilliant successes from thymol in the treatment of ankylostomiasis, so that I can assert that in this disease it often acts like a charm, I must confess that there are some cases-rare, it is true-in which I really found difficulty in ridding the intestines of ankylostoms even by means of this remedy. I now have a case of this kind under treatment, a case which, after all, is surely not one of simple ankylostomiasis. Although in this case the administration three times daily of doses of four grammes brought away in all thirty-one ankylostoms, nevertheless the stools still contain eggs of this parasite in as great abundance as they did previously to the thymol treatment, and as plentifully as they are found in the stools of patients in which thymol brings away hundreds of ankylostoms. I cannot explain on sure grounds the cause of this non-success, but I argue that it may be due to the circumstance that a certain number of worms are still hidden in the walls of the intestine or under the folds of the valvulre conniventes in such a manner that the thymol may pass them without displaying its deadly action on them. It may be also simply that, being enveloped in an extraordinary abundance of tenacious mucus, they are shielded from the action of the drug. These rare cases of non-success must not detract from the reputation of thymol as an admirable remedy in the treatment of ankylostoma; but I wish to insist that its efficacy as against ankylostomiasis cannot in any manner warrant the profession to generalise and to proclaim it as a remedy against all other intestinal parasites without adducing positive proofs of such efficacy in the case of each species of worm. Now I am sure that the only worms I have sometimes seen expelled along with ankylostoma after the administration of thymol are ascaris lumbricoides, oxyuris vermicularis, and trichocephalus diapar; but, while expulsion of ankylostoma is the rule with thymol, this orng acts on the other worms only exceptionally, and in the greater number of cases it altogether fails to effect their expulsion. In this way, for the expulsion of ascaris lumbricoides and oxyuris vermicularis $I$ cannot trust to thymol any more than $I$ can to other ordinary remedies in use, such as santonine (or, better, the new drug, santoninissina) in the case of the former, and the enema of quassia amara or vinegar in that of the latter. As for trichocephalus, it is well known that there is great difficulty in obtaining its expulsion by any remedy. Consequently, after my first trials, in which $I$ found in some cases trichocephali in the stools following the administration of thymol, I entertained the hope that the new drag might prove to be an efficacious remedy against this worm. The ordinary seat of trichocephali being the cæcum I suggested that their expulsion might be assured by using thymol as an enema; but as this means that thymol must be given in solution, I fear that it could rot be used in such doses as to be efficacious as an anthelmintic without proving hurtful to the host by absorption. Indeed, thymol introduced into the circulation is certainly a poison, even in small doses, and if one ventures to give it in such large doses as four, six, eight, and even ten grammes per diem, it is only because it is administered in powders, and it can be relied upon that if ever absorbed it will only be in the minutest proportion. A symptom of dangerous absorption is afforded when the patient presents vertigo or delirium. These unpleasant symptoms are met with only in rare cases after administration of such doses as four grammes of thymol. From what 1 have said, I think that thymol will not prove to be so sure a remedy against the three nematodes named as it is against ankylostoma.

As for the tænia, I have never tried thymol against tænia solium or tænia medio-canellata, because there exists in pelletierine, when properly used, the best known remedy against the large tapeworms; but I can say tbat in cases of ankylostomiasis with tænia nana $I$ did not obtain with thymol the expulsion of the latter. This was brought about only after recourse was had to ethereal extract of male fern, together with calomel. I must add that I have not yet read of well ascertained cases of expulsion of large tapeworms by means of thymol, and I would be grateful to Surgeon-Lieutenant-Colonel Crombie or to any orher practitioner if they would pablish such cases with full particulars, if they know of any. This point is of great importance, because I think it a great therapeutical mistake to consider that a substance which is efficacious in the treatment of one particular parasite must also prove successful as a general 